\title{
Subnanometer-accuracy optical distance ruler based on fluorescence quenching by transparent conductors: supplementary material
}

\author{
Robert J. Moerland* and Jacob P. HoOgenboom* \\ Department of Imaging Physics, Delft University of Technology, NL-2600GA, Delft, The Netherlands \\ *Corresponding author: r.j.moerland@tudelft.nl, j.p.hoogenboom@tudelft.nl
}

Published 21 January 2016

\begin{abstract}
This document provides supplementary information to "Subnanometer-accuracy optical distance ruler based on fluorescence quenching by transparent conductors," http://dx.doi.org/10.1364/optica.3.000112. Transparent conductive oxides (TCOs), such as the well-known indium tinoxide (ITO), find widespread use in modern (nano)technological applications because of their unique combination of negligible optical absorption and good electric conductivity. We, however, show that despite the near-zero imaginary part of the refractive index that is responsible for the material's transparency, TCOs drastically quench optical emitters when the emitter is within $10 \mathrm{~nm}$ of the TCO. Our results reveal that the pure near-field nature of this dissipation makes for an exquisite short-range optical ruler. Previous quenching-based optical rulers, based on interactions with plasmonic or graphene materials, have allowed measuring distances in the 20-100 nm range. Distances below $20 \mathrm{~nm}$ have, however, been hard to assess due to poor photon yields or weak absolute variations. We show that TCO-based rulers close this gap, allowing distance measurements with far-field optics in the 1-10 $\mathrm{nm}$ distance range with deep subnanometer sensitivity. (C) 2016 Optical Society of America
\end{abstract}

http://dx.doi.org/10.1364/optica.3.000112.s001

\section{DETERMINATION OF REFRACTIVE INDICES}

\section{A. Refractive index of ITO}

We use spectroscopic ellipsometry combined with transmittance data at normal incidence to determine the layer thickness and refractive index of the ITO-coated cover glasses used here. The found real and imaginary part of the refractive index are plotted in Fig. S1 as a black and red curve, respectively. The raw spectroscopic data and the settings of their fit can be found in Fig. S2 (separate page).

The wavelength range between $600 \mathrm{~nm}$ and $1000 \mathrm{~nm}$ can be reasonably well approximated by a Drude model [1], where the complex permittivity of the ITO is described by the following equation:

$$
\varepsilon(\omega)=\varepsilon_{\infty}-\frac{\omega_{p}^{2}}{\omega+i \omega \gamma} .
$$

Here, $\varepsilon_{\infty}$ is the permittivity at infinite frequencies, $\omega_{p}$ is the plasma frequency and $\gamma$ is the electron collision frequency. The carrier density $N$ in the ITO and $\omega_{p}$ are related as $\omega_{p}=$ $\left(N e^{2} / \varepsilon_{0} m_{e}^{*}\right)^{1 / 2}$, where $e$ is the magnitude of the elementary charge, $\varepsilon_{0}$ is the permittivity of free space and $m_{e}^{*}$ is the effective mass of electrons $\left(0.4 m_{e}\right.$ for ITO). After fitting, we get $N=6.2 \cdot 10^{20} \mathrm{~cm}^{-} 3$ and $\gamma=4.6 \cdot 10^{13} \mathrm{~s}^{-1}$.

B. Refractive index of $\mathrm{Al}_{2} \mathrm{O}_{3}$

We apply spectroscopic ellipsometry to a $5 \mathrm{~nm}$-thick layer of $\mathrm{Al}_{2} \mathrm{O}_{3}$, deposited on a silicon wafer by Atomic Layer Deposition (ALD). We find that the refractive index of the $\mathrm{Al}_{2} \mathrm{O}_{3}$ layer can be described well with the Cauchy relation $n(\lambda)=$ $A+B / \lambda^{2}+C / \lambda^{4}$, with $A, B$ and $C$ constants and $\lambda$ the wavelength in micrometer. Here, $A=1.751, B=0.00632$ and $C=-0.00010152$. This relation assumes a lossless dielectric 
Fit: \begin{tabular}{|lll}
\hline Generate & Fit & Fit Dynamic \\
\end{tabular}

MSE $=2.383$

Roughness $=0.00 \pm 0.896 \mathrm{~nm}$

Thickness \# $1=17.34 \pm 1.029 \mathrm{~nm}$

Einf $=2.210 \pm 0.1817$

UV Pole Amp. $=8.9366 \pm 6.11339$

UV Pole En. $=5.352 \pm 0.6076$

IR Pole Amp. $=1.6920 \pm 61.13246$

Resistivity $(\mathrm{Ohm} \cdot \mathrm{cm}) 2=0.002953 \pm 3.3036692$

Scat. Time (fs) $2=15.158 \pm 8497.0035$

$\mathrm{k}$ Amplitude $=1.5580 \mathrm{E}-06 \pm 1.0770 \mathrm{E}-06$
Layer Commands: Add Delete Save

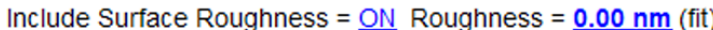

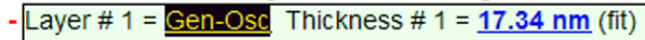

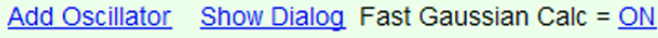

$\operatorname{Einf}=\underline{\mathbf{2 . 2 1 0}}$ (fit)

UV Pole Amp. $=\underline{\mathbf{8 . 9 3 6 6}}$ (fit) UV Pole En. $=\underline{\mathbf{5 . 3 5 2}}$ (fit)

IR Pole Amp. $=\underline{1.6920}$ (fit)

Fit All Clear All Add Amp. Add Br. Add En.

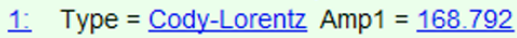

$\mathrm{Br} 1=\underline{2.176} \mathrm{E} 01=\underline{4.298} \mathrm{Eg} 1=\underline{3.415}$

$\mathrm{Ep} 1=\underline{7.439} \mathrm{Et} 1=\underline{0.00} \mathrm{Eu} 1=\underline{0.389} \mathrm{Common} \mathrm{Eg}=\underline{\mathrm{OFF}}$

2. Type $=\underline{\operatorname{Drude}(\mathrm{RT})}$ Resistivity $(\mathrm{Ohm} \cdot \mathrm{cm}) 2=\underline{\mathbf{0 . 0 0 2 9 5 3}}$ (fit) Scat. Time (fs)2 $=\underline{15.158}$ (fit) - Substrate $=$ 7059 Cauchy

$A=\underline{1.508} B=\underline{0.00510} C=\underline{0.0000}$

$\mathrm{k}$ Amplitude $=\underline{1.5580 \mathrm{E}-06}$ (fit) Exponent $=\underline{4.270}$

Band Edge $=\underline{350.0 \mathrm{~nm}}$

Angle Offset $=\underline{0.00}$

Variable Angle Spectroscopic Ellipsometric (VASE) Data
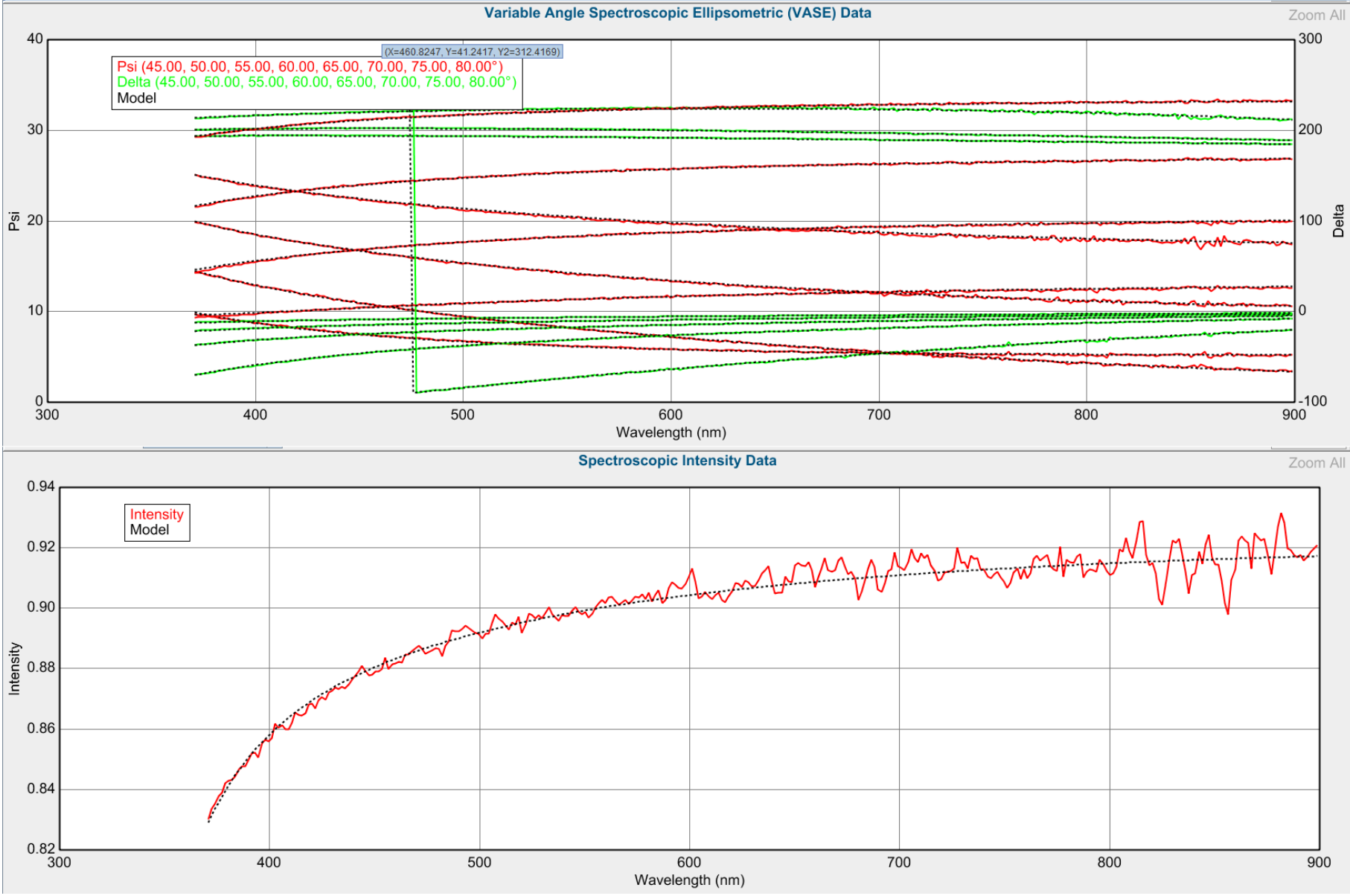

Fig. S2. Settings of and fit obtained with spectroscopic ellipsometry, including transmittance data. 


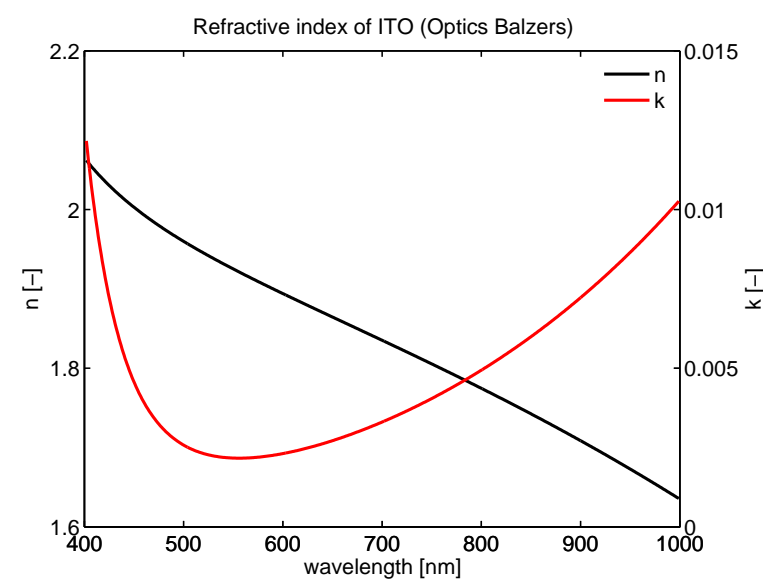

Fig. S1. Measured complex refractive index of ITO by means of spectroscopic ellipsometry combined with transmittance data. See also Fig. S2 for settings.

$(k=0)$, an approximation that holds for our experiment, since our data implies $k_{\mathrm{Al}_{2} \mathrm{O}_{3}} \ll k_{I T O}$. If the latter would not be true, we would not be able to measure an increase in lifetime (and vice versa, lower losses) with increasing distance to the ITO layer.

\section{CALCULATED REFLECTANCE, TRANSMITTANCE AND ABSORBANCE}

We use the experimentally obtained refractive index of ITO (see Supplement 1, Section 1) to calculate the reflectance, transmittance and absorbance of a $17 \mathrm{~nm}$-thick layer of ITO on a glass substrate. The results are shown in Fig. S3 for light in the $400 \mathrm{~nm}$ to $800 \mathrm{~nm}$ range, at normal incidence. For clarity, the absorbance is shown after multiplication by a factor 100 .

\section{SURFACE ROUGHNESS OF ITO AND $\mathrm{AL}_{2} \mathrm{O}_{3}$}

The description of dipole radiation by the angular spectrum representation[2-5] assumes perfectly planar and smooth surfaces. Here we show that the ITO-coated glass substrates (Optics Balzers) used here approximate the theoretical situation to a sufficient degree. In Fig. S4, Atomic Force Microscopy (AFM) images are shown of a bare ITO slide (top) and an ITO slide coated with $5 \mathrm{~nm}$ of $\mathrm{Al}_{2} \mathrm{O}_{3}$, with Tetraphenoxy-Perylene Diimide molecules applied to it (bottom). The AFM scans were analyzed with the open-source software package Gwyddion [6]. Before analysis, a polynomial background subtraction of order 2 was performed on the data to reduce scanning artifacts.

The ITO coating shows, in a $2 \times 2 \mu \mathrm{m}$ area, a height distribution with a standard deviation of $0.4 \mathrm{~nm}$. This minor thickness variation does not directly influence the outcome of the measurement, as is also shown in Fig. 6 in the main text. After performing the ALD step, the standard deviation is $0.5 \mathrm{~nm}$. More importantly, on the molecular scale, the local flatness is lower. Fig. S5 shows a line profile taken across the AFM data for the bare ITO slide, shown in Fig. S5a, and for the $\mathrm{Al}_{2} \mathrm{O}_{3}$ coated slide, shown in Fig. S5b. The profiles correspond to the red lines in Fig. S4. Spatial oscillations with periods longer than $390 \mathrm{~nm}$ are attributed to local curvature of the surface and not surface roughness, which is reflected in Fig. S5 by introducing the waviness and roughness of the surface. Note that this is a

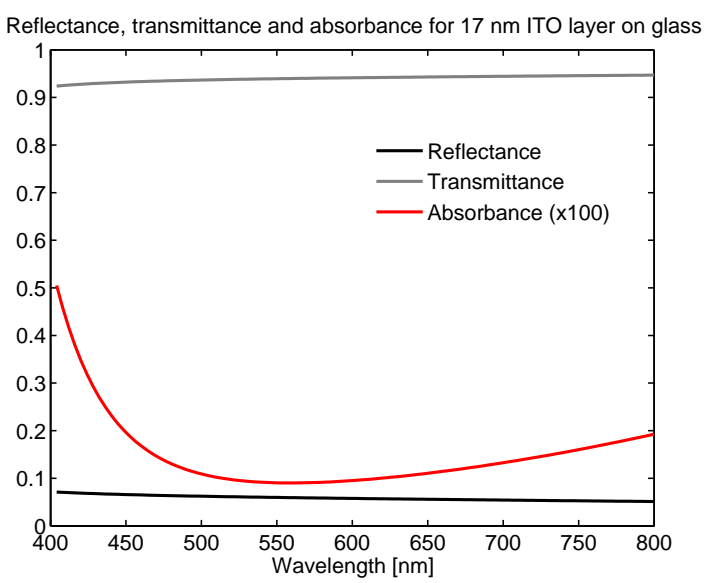

Fig. S3. Calculated reflectance, transmittance and absorbance at normal incidence for a glass substrate covered with a $17 \mathrm{~nm}$ thick layer of ITO with a refractive index as shown in Fig. S1. Note that the absorbance is scaled by a factor of 100, meaning that over the whole displayed wavelength range the absorbance is less than $1 \%$.

conservative estimate of the roughness, as only roughness on the scale below some tens of nanometers could strongly influence the results. A roughness analysis of the profiles yields a root-mean-square roughness of $0.15 \mathrm{~nm}$ for the bare ITO slide and $0.20 \mathrm{~nm}$ for the ITO slide after ALD processing. Therefore, we conclude that the variation of the height of the ITO layer is sufficiently low to approximate the surface as flat. Moreover, the ALD step adds negligibly to the surface roughness, thus the ALD-deposited $\mathrm{Al}_{2} \mathrm{O}_{3}$ can be considered smooth, even when compared to the steps of $0.33 \mathrm{~nm}$ in the main text.

\section{FIT OF LIFETIME DATA TO THE THEORETICAL MODEL FOR TETRAPHENOXY-PERYLENE DIIMIDE}

For the theoretical prediction of the lifetime of TetraphenoxyPerylene Diimide versus alumina spacer thickness (solid curve in Fig. 2b in the main text), we use Eq. 1 from the main text. For $\tau_{0}$, the lifetime of the molecule in vacuum, we use the measured lifetime of the molecule on glass (6.1 ns) multiplied with the relative local density of states of the molecule on glass (1.4), yielding $\tau_{0}=8.6 \mathrm{~ns}$. Thus, we ignore the potential presence of additional non-radiative pathways such as quenching by oxygen, and we assume a QY of $100 \%$ for the molecules in vacuum. The benefit is that with this approximation, there is only one unknown in the model, which is the effective height of the molecular transition dipole with respect to the $\mathrm{Al}_{2} \mathrm{O}_{3}$ layer. $\mathrm{A}$ fit to the measured data yields a height of $1.13 \pm 0.05 \mathrm{~nm}$. The inset in Fig. $2 \mathrm{~b}$ in the main text shows the measured mean fluorescence intensity as a function of the spacer layer thickness. Here, we forego additional fitting and equate the measured normalized intensity at a spacer thickness of $10 \mathrm{~nm}$ to the QY as predicted by the model. The remaining intensity measurements are scaled accordingly.

\section{FIT OF LIFETIME DATA TO THE THEORETICAL MODEL FOR ALEXA FLUOR 430}

For the case of Alexa Fluor 430, the assumption that the QY is $100 \%$ does not reproduce the experimental data. Instead, we 

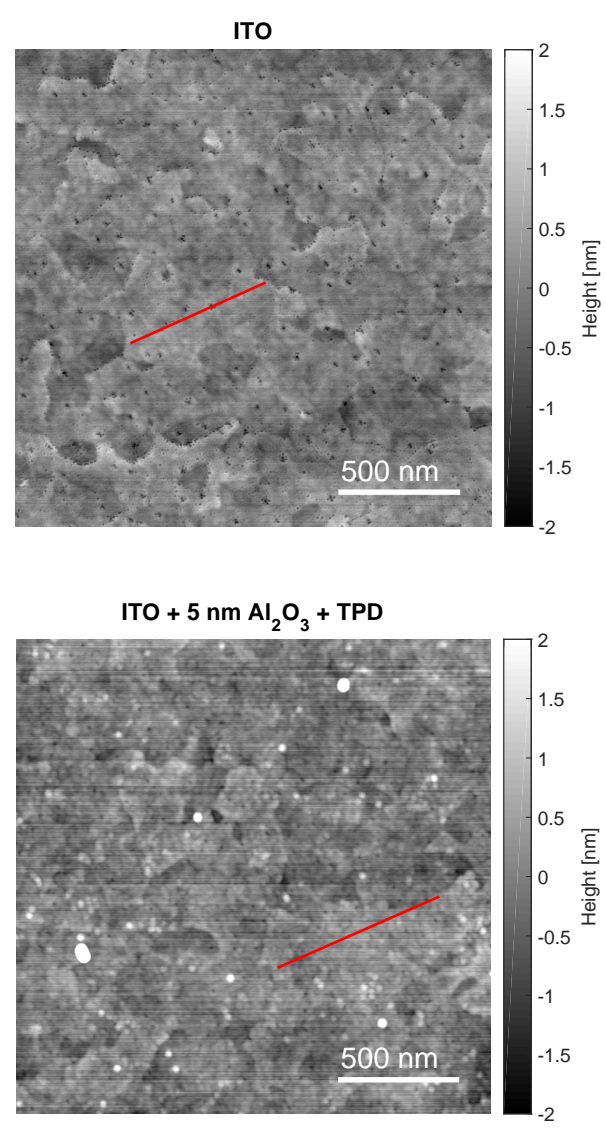

Fig. S4. Atomic Force Microscopy (AFM) image of an ITOcoated glass substrate before (top) and after (bottom) adding an additional layer of $\mathrm{Al}_{2} \mathrm{O}_{3}$ by ALD. In this particular case, the $\mathrm{Al}_{2} \mathrm{O}_{3}$ is $5 \mathrm{~nm}$ thick. In both AFM images, the imaged area is $2 \times 2 \mu \mathrm{m}$ large. The height distribution has a standard deviation of $0.4 \mathrm{~nm}$ for the bare subtrate, whereas after the ALD step this is $0.5 \mathrm{~nm}$.

estimate the intrinsic quantum yield to be about 0.2 based on a fit with the model [7] $\gamma=\gamma_{i}+\gamma_{r} \cdot \operatorname{LDOS}(d, h)$. Here, $\gamma_{i}$ and $\gamma_{r}$ are the non-radiative rate intrinsic to the molecule and the radiative rate of the emitter in vacuum, respectively. LDOS represents the local density of states, which depends on the thickness of the $\mathrm{Al}_{2} \mathrm{O}_{3}$ layer $d$ and the effective height $h$ of the molecule above this layer. As an additional data point we use the lifetime of the molecule on a glass substrate with $2 \mathrm{~nm}$ of alumina applied to it, without ITO (2.59 ns). We find $=0.3, \gamma_{r}=0.06$ and $h=0.54 \mathrm{~nm}$ through fitting.

\section{CALCULATIONS OF LIFETIME AND QUANTUM YIELD FOR A VERTICALLY ORIENTED DIPOLE}

The main text discusses the reduction of lifetime and quantum yield for horizontal dipoles. We claim that vertically oriented dipoles show similar behavior when the lifetime and quantum yield is compared for substrates that are either bare or coated with silver and ITO, for distances less than about $10 \mathrm{~nm}$. For these small distances, the dipole field is dominated by the near field, which displays an $r^{-3}$ dependency regardless of dipole orientation $[5,8]$. Therefore, vertically oriented dipoles show the same distance dependency for this type of quenching as hor-
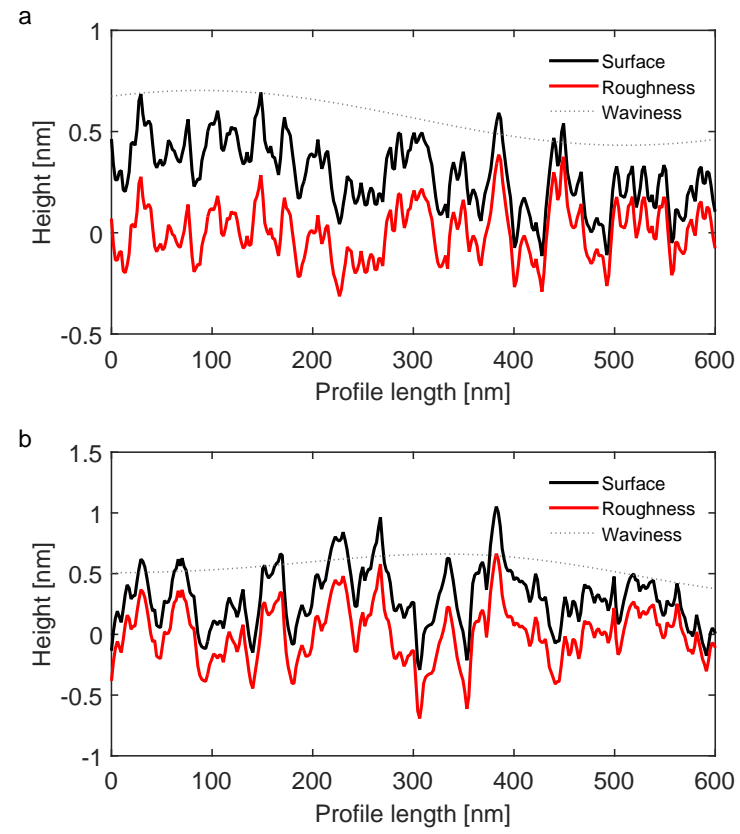

Fig. S5. Height profiles taken along the AFM data shown in Fig. S4. In (a), the profile of a bare ITO slide is shown, whereas in (b), the profile of an ITO slide after ALD processing is shown. In this particular case, $5 \mathrm{~nm}$ of $\mathrm{Al}_{2} \mathrm{O}_{3}$ was added on top of the ITO. The black solid curves show the raw height data. Spatial oscillations with periods longer than $390 \mathrm{~nm}$ are attributed to a local curvature of the surface and not surface roughness, by introducing the waviness (grey dotted curve) and roughness (red curve) of the surface. For the bare substrate, a root-mean-square roughness of $0.15 \mathrm{~nm}$ is found. After ALD processing, the surface roughness is found to be $0.20 \mathrm{~nm}$. Therefore, the impact of ALD on the roughness of the surface is minimal, and the roughness is much less than the height steps taken in the main text.

izontal dipoles, but the onset of the near-field quenching might differ slightly due to the different orientations. The lifetimes at the onset of quenching will in general be different for horizontal and vertical dipoles. Figure $\mathrm{S} 6$ shows the result of a calculation of the lifetime and quantum yield for vertically oriented dipoles, as a function of distance, confirming our statement.

The plausibility of our approximation, where we only take horizontal dipoles into account, stems from a few arguments. First of all, the polarization in the focus of our objective, even for a high-NA objective, is still dominated by the polarization state of the excitation laser [5]. Therefore the excitation polarization is strongly horizontal at the sample plane. Secondly, the excitation efficiency of molecules scales as $|\mathbf{E} \cdot \mathbf{p}|^{2}$, with $\mathbf{E}$ the local electric field and $\mathbf{p}$ the dipole orientation. Therefore, the excitation efficiency is the highest for horizontal molecules, and drops with $\cos ^{2} \phi$, where $\phi$ is the angle between electric field and the dipole orientation. Combined, this means that we most efficiently excite molecules with a strongly horizontal dipole orientation. If our set of excited molecules would contain a substantial amount of vertically-oriented dipoles, clear non-single-exponential lifetime data would show the mixing of the two orientations, as they have different lifetimes. The fact that our measured life- 

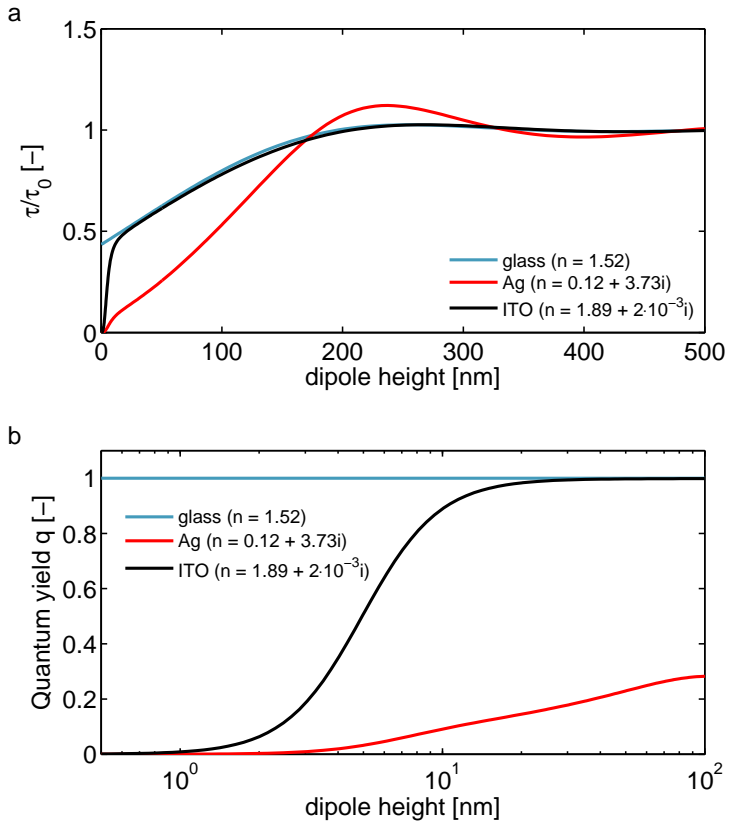

Fig. S6. Predicted variations in lifetime (a) and quantum yield (b) for a vertically oriented dipole emitter.

time data shows reasonable single-exponential behavior implies that indeed we mostly probe horizontal dipoles. Nevertheless, dipoles that are not perfectly horizontal still will show the same near-field quenching behavior, as shown above, and therefore are equally suitable for ruler applications.

\section{REFERENCES}

1. M. D. Losego, A. Y. Efremenko, C. L. Rhodes, M. G. Cerruti, S. Franzen, and J.-P. Maria, "Conductive oxide thin films: Model systems for understanding and controlling surface plasmon resonance," J. Appl. Phys. 106, 024903 (2009).

2. H. Weyl, "Ausbreitung elektromagnetischer wellen über einem ebenen leiter," Ann. Phys. 365, 481-500 (1919).

3. W. Lukosz and R. E. Kunz, "Light-emission by magnetic and electric dipoles close to a plane interface. i. total radiated power," J. Opt. Soc. Am. 67, 1607-1615 (1977).

4. L. Novotny, "Allowed and forbidden light in near-field optics. i. a single dipolar light source," J. Opt. Soc. Am. A 14, 91-104 (1997).

5. L. Novotny and B. Hecht, Principles of Nano-Optics (Cambridge University Press, Cambridge, 2006), 1st ed.

6. Gwyddion. http://gwyddion.net/.

7. B. Buchler, T. Kalkbrenner, C. Hettich, and V. Sandoghdar, "Measuring the quantum efficiency of the optical emission of single radiating dipoles using a scanning mirror," Phys. Rev. Lett. 95, 063003 (2005).

8. W. L. Barnes, "Fluorescence near interfaces: The role of photonic mode density," J. Mod. Opt. 45, 661-699 (1998). 